\title{
Field Tests of the Vertical-Array Differential Target Antenna Coupling (DTAC) System
}

\author{
Wanjie Feng* and Ben K. Sternberg \\ Laboratory for Advanced Subsurface Imaging (LASI), University of Arizona, 1235 E. James E. Rogers Way Tucson, \\ AZ 85721-0012 \\ bkslasi@email.arizona.edu \\ wanjie.feng@zonge.com
}

*Now at Zonge International, 3322 E. Fort Lowell Rd, Tucson, AZ 85716

\begin{abstract}
We have tested the Vertical-Array Differential Target Antenna Coupling (DTAC) system over a variety of well-characterized, buried targets. The DTAC system produces a primary field in the frequency range of $10 \mathrm{~Hz}$ to $10 \mathrm{kHz}$ and records secondary magnetic fields from subsurface targets, relative to a reference frequency. The current DTAC system has higher power, compared with our earlier tests, and this system is suitable for either ground or airborne mapping of a wide variety of near-surface targets, which might be encountered in civil engineering, water resources, environmental characterization, mining, and other natural-resource exploration. Profiles over three well-characterized targets show a close agreement between the measured DTAC response and model simulations. Conventional field measurements using induced magnetic fields are also displayed and they do not show nearly as effective mapping of the targets. Another profile shows that the DTAC method has greatly reduced sensitivity to surface clutter, compared to conventional measurements.
\end{abstract}

\section{Introduction}

Previous numerical modeling studies and smallscale prototype measurements have shown that the Differential Target Antenna Coupling (DTAC) method for electromagnetic imaging of the subsurface has the potential to detect targets deeper and with greater resolution of target dip, strike, and thickness, compared with conventional EM methods (Sternberg et al., 2015a,b). The DTAC method uses at least two frequencies in the measurement scheme. One frequency is used as a reference signal to establish the null direction at that frequency and then a different frequency is used to measure the change in the null at that new frequency. It can also use an arbitrary number of frequencies with just one reference frequency for wide-bandwidth measurements.

\section{Horizontal-Array DTAC Method}

The theory behind the horizontal-array DTAC method was described in Dvorak and Sternberg (2013), and this paper also showed numerical examples of DTAC responses for large, deeply-buried targets. A second paper
(Sternberg and Dvorak, 2013) provided an experimental verification of the DTAC system's measurement capabilities. To demonstrate the accuracy of the DTAC method, the papers showed both measured and calculated responses for a variety of precisely known, small-scale targets. In these papers, the DTAC system employed a fixed TX loop and a single RX coil that was moved along a profile line. Because of the horizontal separation between the fixed TX and moving RX, this technique will be referred to as the horizontal-array DTAC method. In this method, a single RX coil, which is mounted at an angle on a rotation axis, is rotated to three angular orientations at each measurement location, and the corresponding components of the magnetic field are measured in these three orientations at multiple frequencies. These measured fields are then used to back-calculate the true $x, y$, and $z$ components of the magnetic field at each of these frequencies. The technique uses the back-calculated components of the magnetic field to numerically determine the null orientation for the total vector field response (i.e., the primary response, the secondary earth response, and the target response) at a reference frequency and multiple data frequencies. If there is no target present, then 
Journal of Environmental and Engineering Geophysics

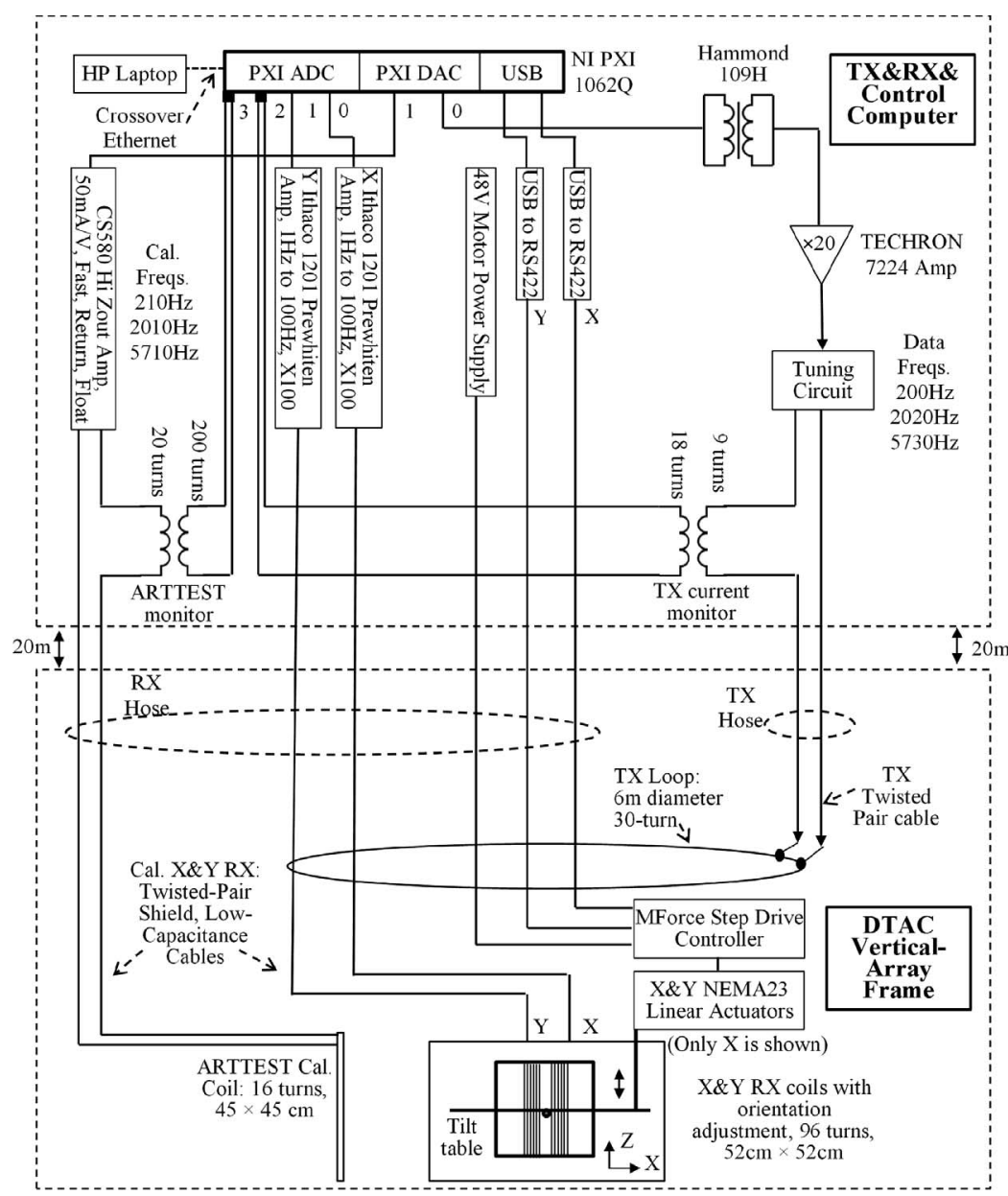

Figure 1. DTAC high-power system block diagram.

the null will appear in the same orientation for all frequencies, and thus there will be no DTAC response at any of the frequencies. On the other hand, if a target is present, then the null orientation will change with frequency, and the RX coil will pick up the small secondary field response associated with the buried target.

\section{Vertical-Array DTAC Method}

While application of the DTAC method to a horizontal array is well suited for imaging deep targets, it can be time consuming to carry out measurements since a large, fixed TX loop must be laid out prior to conducting a survey. To allow for more rapid measurements, it is desirable to employ a more compact vertical array where both the TX and RX can be moved simultaneously along the profile line. This vertical array could be either towed by a vehicle or it could be suspended below an aerial platform, e.g., a helicopter.

To investigate the feasibility of such a system, Sternberg et al. (2015a) studied the extension of the
DTAC method to a moving, vertical TX/RX array, using a very low-power system over known tuned-coil targets. This null-field array utilizes a horizontal TX loop and an approximately orthogonal (nulled) two-axis RX that is located directly below the TX. Both the TX and RX coils are fixed to a frame, which can be moved by a cart, vehicle, or helicopter. Dvorak and Sternberg (2014) describe the theoretical basis for the vertical-array DTAC method and then employed this analysis along with numerical examples to explain the physical phenomenology behind the vertical-array DTAC method. Sternberg et al. (2015b) show numerical modeling of the DTAC response for a wide variety of geologic targets.

\section{Objective of This Paper}

In this paper we will concentrate on the verticalarray DTAC method, because of its suitability for either ground or airborne mapping of a wide variety of nearsurface targets that might be encountered in civil engineering, water resources, environmental character- 


\section{Feng and Sternberg: DTAC System Field Tests}

ization, mining, and other natural resource exploration. Sternberg et al. (2015a) demonstrated some of the capabilities of the vertical-array DTAC method using very small-scale surveys and using artificial tuned-target coils. In this paper, we will expand the field testing of the DTAC method to more realistic targets. We have chosen to use known targets, buried at relatively shallow depths, during this phase of our research. This was necessary because we wanted to verify the details of the measured response, and this can only be done with a precisely known target. A future paper will address the application of the DTAC method to subsurface geologic targets, for which the target characteristics may be known based only on limited drill-hole information.

\section{The DTAC High-Power Data-Acquisition System}

Figure 1 shows a block diagram of the current DTAC High-Power Data-Acquisition System. A signal with three frequencies from a digital-to-analog converter is used to drive a Techron high-power linear amplifier. This signal then goes to a tuning circuit, which is described in Dvorak et al. (2017). The Tuning Circuit signal output is monitored by an analog to digital converter and transmitted to a horizontal-loop transmitting antenna, $6 \mathrm{~m}$ diameter, with 30 turns. In the current DTAC system, the currents are $4 \mathrm{~A}, 4 \mathrm{~A}$, and $2 \mathrm{~A}$ at 200 $\mathrm{Hz}, 2,020 \mathrm{~Hz}$, and $5,730 \mathrm{~Hz}$ respectively. The design of the transmitter coil is described in Sternberg et al. (2017). The two receiver coils $\left(B_{x}\right.$ and $\left.B_{y}\right)$ are in approximate null-coupling to the transmitter-loop primary field. Dvorak and Sternberg (2014) showed that a theoretical $\mathrm{B}_{\mathrm{z}}$ field can be used in the DTAC calculations and it is not necessary to measure $B_{z}$ with the vertical array. A linear actuator, controlled by the computer, is used to adjust a tilt table for the best null-coupling to the transmitter primary field. Signals from the $\mathrm{X}$ and $\mathrm{Y}$ receiver coils pass through a pre-whitening amplifier to an analog-to-digital converter. A calibration signal is used to provide a continuous calibration simultaneously with data acquisition. This simultaneous calibration is known as the ARTTEST calibration (Sternberg and Dvorak, 2002; Dvorak and Sternberg, 2002).

Figure 2(a) shows the DTAC survey system frame, which contains the receiver coils at the bottom of the frame, and the 30 turn, $6 \mathrm{~m}$ diameter transmitting loop is at the top of the frame. Figure 2(b) shows the DTAC tow truck which is used to pull the DTAC frame on a small cart. The truck also contains an AC power generator, the Techron high-power linear amplifier, the tuning circuit, and the data-acquisition system.

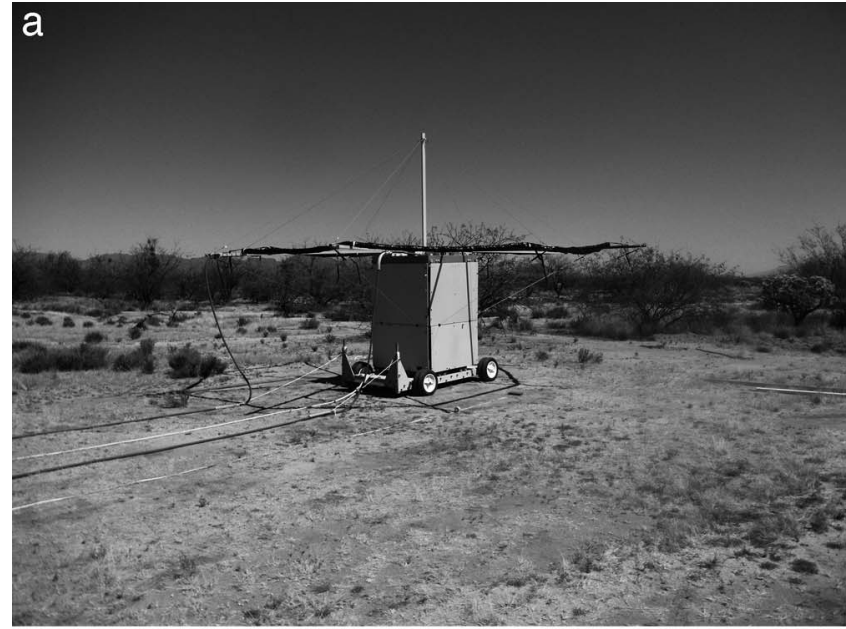

b

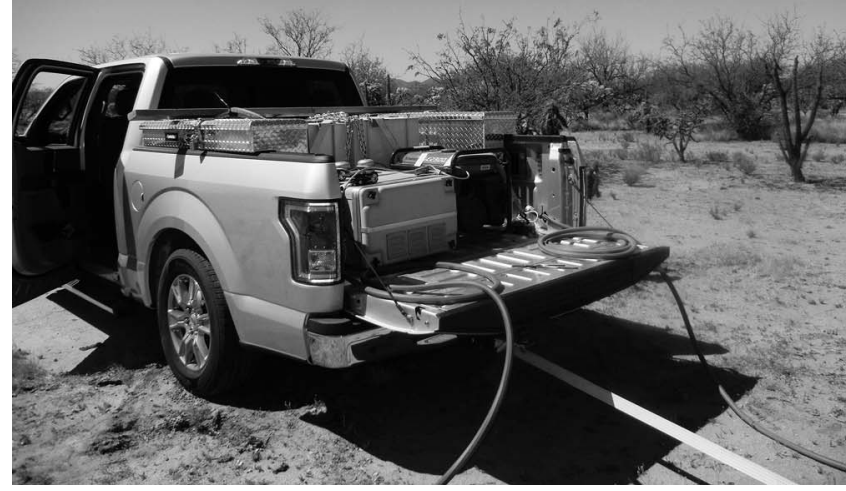

Figure 2. DTAC survey system. a) DTAC frame, and $6 \mathrm{~m}$ diameter, 30-turn transmitting loop; b) DTAC tow truck, with generator, transmitter, and tuning circuit.

In this paper, we are only measuring the $\mathrm{B}_{\mathrm{x}}$ and $\mathrm{B}_{\mathrm{y}}$ responses, since they are the most important components for imaging the 3D targets we have chosen for the field tests. The DTAC frame, shown in Fig. 2(a), can also be used to measure the $B_{z}$ field, which is very useful for mapping the layered-earth resistivity. $B_{z}$ is measured by placing a horizontal receiver coil at a location where the $\mathrm{B}_{\mathrm{z}}$ field is in null coupling to the transmitter loop, for example at a location that is offset from the center of the transmitting loop and below the plane of the loop. At this location, the $\mathrm{B}_{\mathrm{z}}$ field can effectively map the layered-earth resistivity.

\section{Three-Meter-Deep Sheet Target}

The first target, located at our local test site, is an aluminum sheet $0.6 \mathrm{~m}$ wide, $3.2 \mathrm{~m}$ long, $3.1 \mathrm{~mm}$ thick, buried horizontally, at a depth of $3 \mathrm{~m}$. The profile is 
(a)

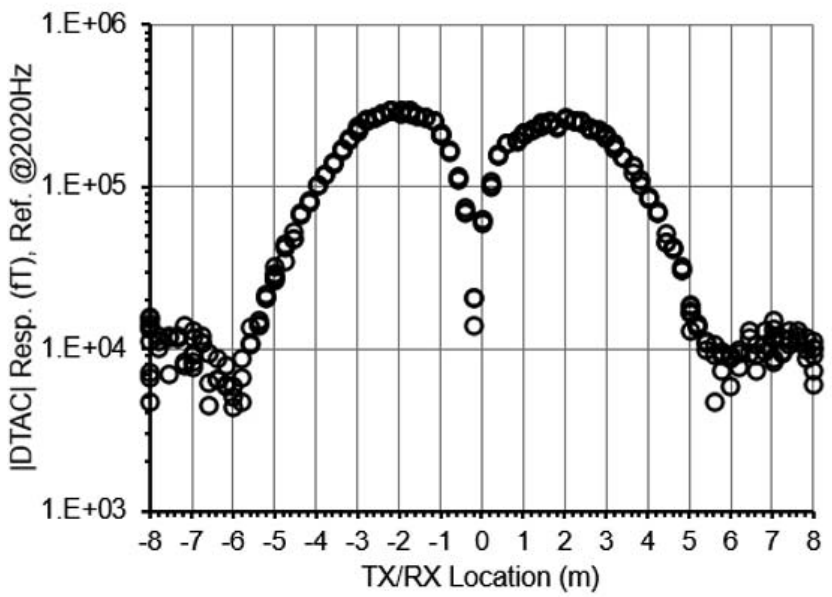

(c)

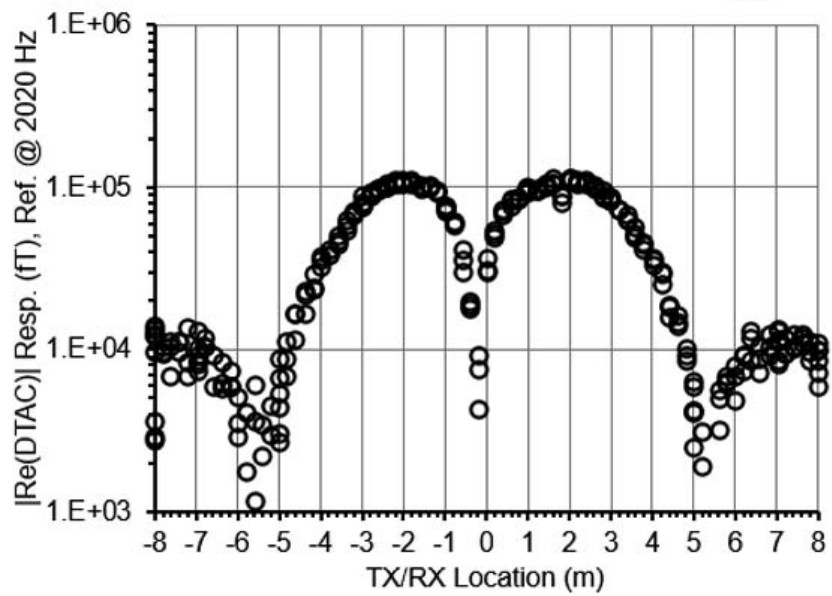

(b)

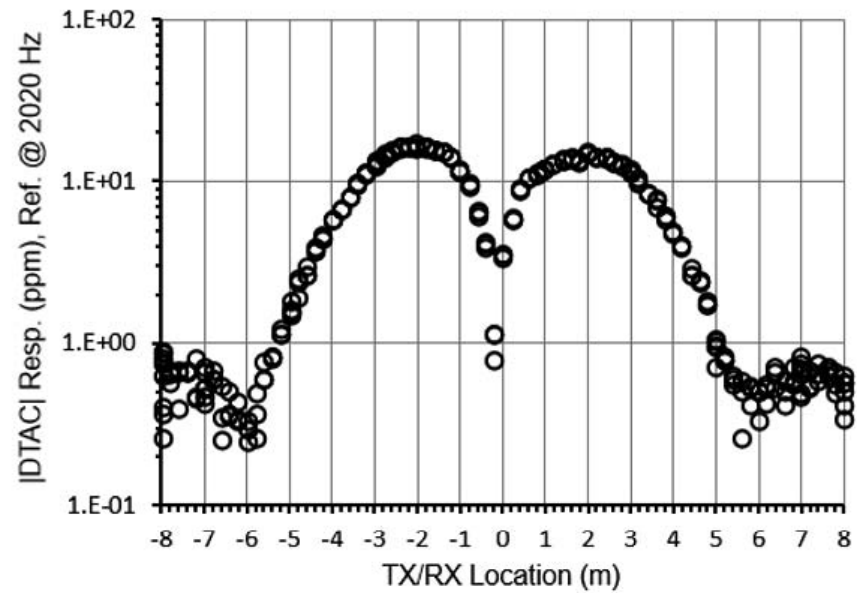

(d)

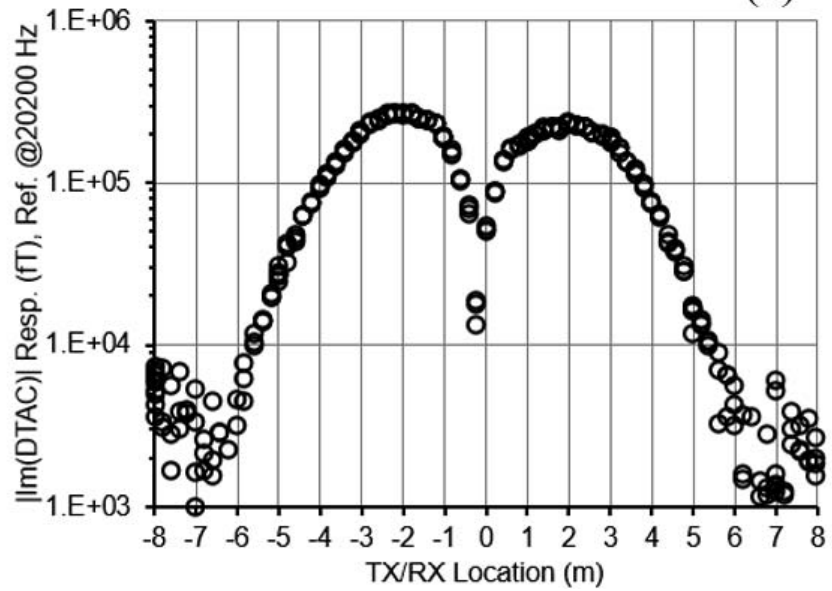

Figure 3. DTAC data from a three-meter-deep sheet. The reference frequency is $2020 \mathrm{~Hz}$ and data frequency is 200Hz. a) DTAC magnitude in fT; b) DTAC magnitude in ppm; c) Real DTAC responses in fT; d) Imaginary DTAC responses in $\mathrm{fT}$.

perpendicular to the sheet and $0.6 \mathrm{~m}$ from the east edge of the sheet. This simple sheet is very useful for testing the system and debugging the electronics and data-processing procedures. Figure 3 shows the results of a DTAC profile over this shallow target. On the bottom of this figure, we show the real and imaginary DTAC response. At the top, we show the calculated magnitude in femtoteslas (fT), and the same calculated magnitude in parts per million (ppm) of the primary field. For this test, we have removed a constant response from a broad geologic background anomaly. The profile response shows a very clear symmetric response, and with low background noise levels of several thousand fT, which is the expected background EM noise level in this region during the late summer monsoon season when these data were collected.
In Fig. 4, we show a theoretical calculation of the expected sheet response, using the EMIGMA V8.6 modeling program (EMIGMA, 2016). We see that there is a very close match between the measured response and the theoretical model response. This includes all details, such as the peak magnitude and the symmetrical features at the edges of the anomaly. For the EMIGMA calculation, we used the VHPLATE algorithm (Walker and West, 1991), with a conductivity-thickness product of 50,000 S, a depth of $3 \mathrm{~m}$, buried in an $80 \mathrm{Ohm}-\mathrm{m}$ half space (based on our previous research at this site). The parameters of the model and the profile offset relative to the model are the same as the actual buried aluminum sheet.

We note that we handle the EMIGMA theoretical data in exactly the same way that we handle the observed data. In other words, we find the null field at the 
Feng and Sternberg: DTAC System Field Tests

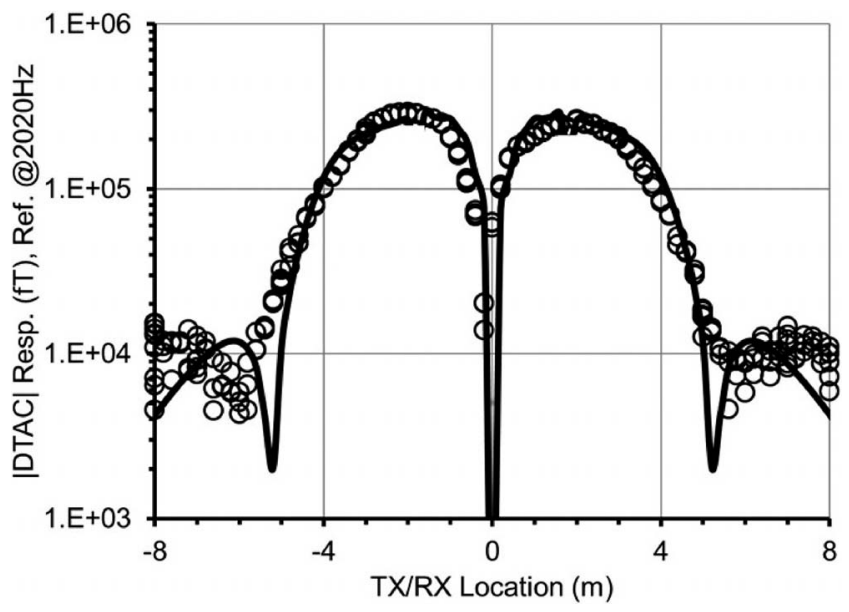

Figure 4. Three-meter-deep sheet DTAC data with theoretical calculation of a line for EMIGMA VHPLATE response. The reference frequency is $2,020 \mathrm{~Hz}$ and data frequency is $200 \mathrm{~Hz}$.

reference frequency, and then calculate the response at each data frequency, for both the measured and the theoretically modeled data. The DTAC processing therefore does not introduce any distortion in the data, but does provide significant advantages in terms of data stability and resolving power.

\section{Three Steel-Barrel Targets}

For our next test, we looked at a set of relatively small targets buried at a range of depths. The steel barrel is a standard 55-gallon drum, and is $0.6 \mathrm{~m}$ in diameter and $0.8 \mathrm{~m}$ long. The barrel was buried with the long side horizontal and north-south. The distance locations and the depths of the barrels are plotted at the center of the barrel. The first barrel is located at $14.5 \mathrm{~m}$ on the profile and is at a depth of $2.2 \mathrm{~m}$; the second barrel is located at a distance of $35.3 \mathrm{~m}$ and is at a depth of $3.4 \mathrm{~m}$; the third barrel is at $55 \mathrm{~m}$ and is at a depth of $4.8 \mathrm{~m}$. In this test, the profile was along the south edge of the barrels from west to east.

We found that for this data set, the imaginary or quadrature response was much noisier than the real or inphase response. We have therefore used only the inphase data. The quadrature field values were set to very small constant values along the entire profile, e.g., 100 fT. Note that the quadrature values cannot be set to zero, or there will be no DTAC response. However, if they are set to a very small value, they have little effect on the DTAC response. With this approach we are still finding a null field at a reference frequency and measuring data frequencies to find the DTAC response.

The barrel response shows the expected simple progression from large to medium to small anomalies as we proceed from shallow to medium to large depth, as shown in Fig. 5. The background noise level is again in the 1,000 to $10,000 \mathrm{fT}$ range, which is what we would expect in the late summer monsoon season in the southwestern U.S.

Figure 6 shows the DTAC response in fT, along with the theoretically calculated DTAC response for these barrels (as shown by the solid line). For the EMIGMA calculation in this example we have used a prism model and the Inductive Localized Nonlinear (ILN) algorithm (Murray, 1997; Murray et al., 1999), with a background resistivity of $50 \mathrm{Ohm}-\mathrm{m}$, the prism has a volume equal to the barrel volume, and the prism has a conductivity of $6,000 \mathrm{~S} / \mathrm{m}$. We note that the 6,000

(a)
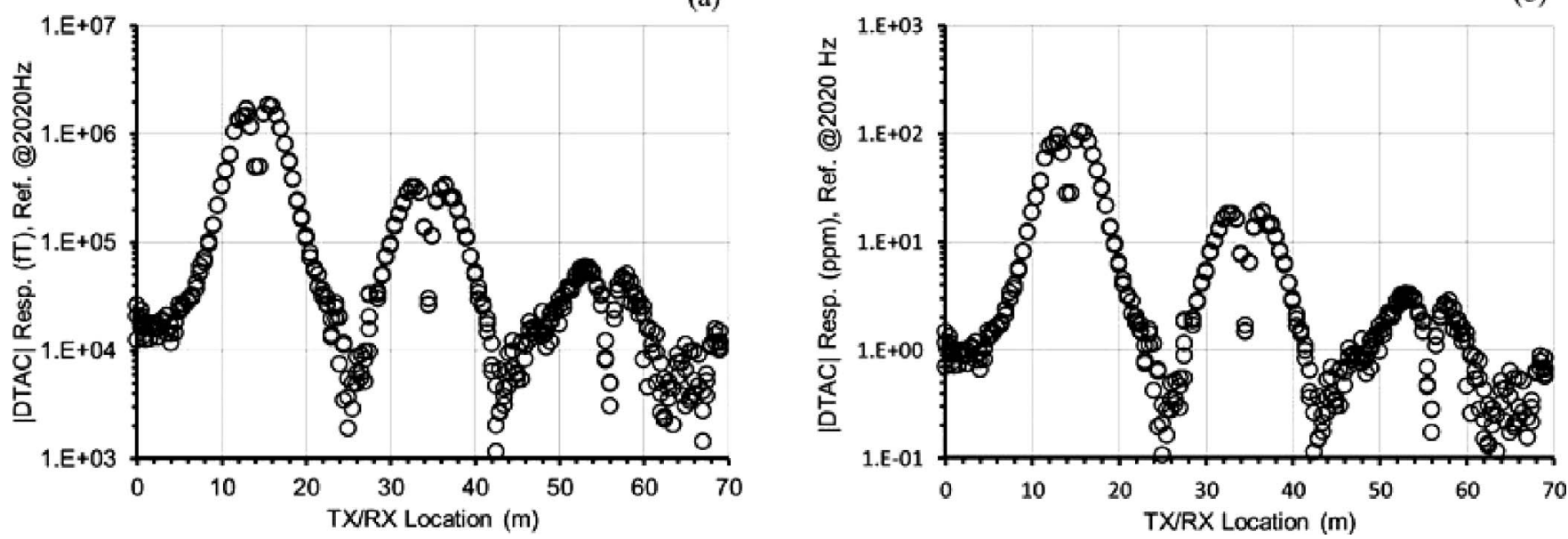

(b)

Figure 5. DTAC profiles over three steel barrels at depths of $2.2 \mathrm{~m}$ at $14.5 \mathrm{~m}, 3.4 \mathrm{~m}$ at $35.3 \mathrm{~m}$, and $4.8 \mathrm{~m}$ at 55 $\mathrm{m}$. The reference frequency is $2,020 \mathrm{~Hz}$ and data frequency is $200 \mathrm{~Hz}$. a) The DTAC magnitude responses in fT; b) the DTAC magnitude responses in ppm. 
Journal of Environmental and Engineering Geophysics

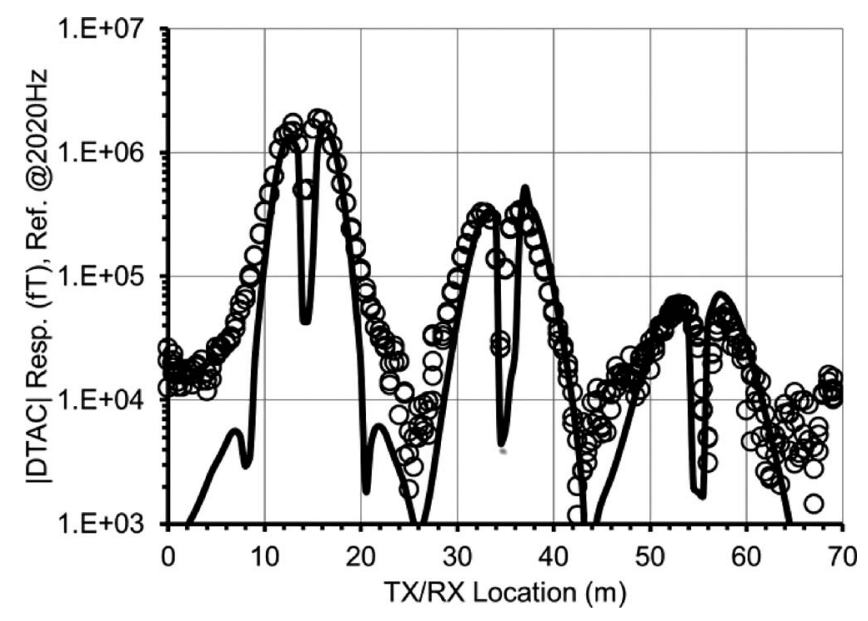

Figure 6. Three-barrel profile with theoretical calculation (line) for EMIGMA ILN Prism response. The reference frequency is $2,020 \mathrm{~Hz}$ and data frequency is $200 \mathrm{~Hz}$.
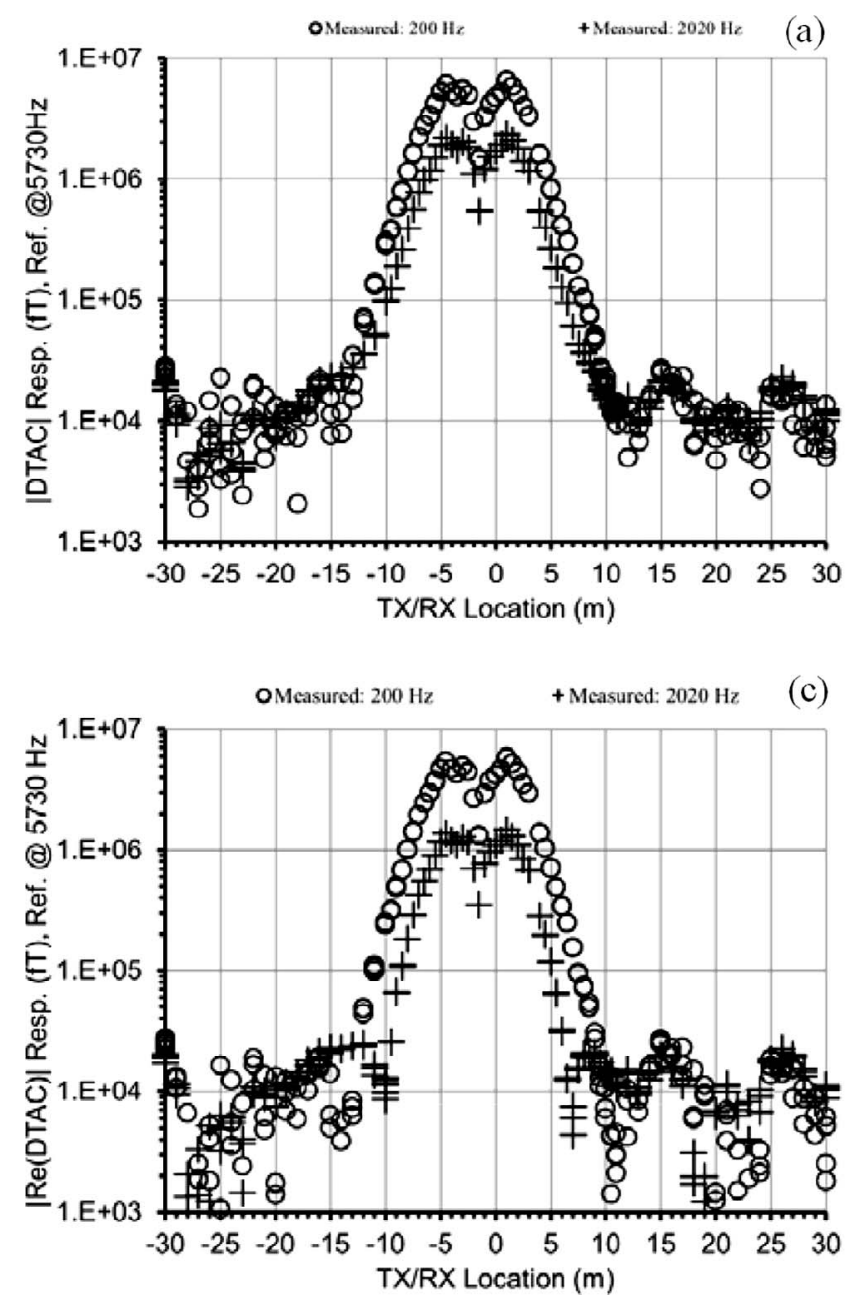

$\mathrm{S} / \mathrm{m}$ value is a composite value for the steel barrel and the air-filled cavity inside the barrel. Once again, there is a very close match between the measured response and the theoretical model response.

We note that the widths of the three DTAC anomalies in Fig. 6 are substantially different. The shallow anomaly is narrow, and the deeper anomalies are progressively wider, as we would expect. The distinctive features of the DTAC responses allow us to quantitatively model the measured responses and determine target size, shape, and depth. The DTAC method is far more than just an anomaly finder.

\section{Long Concrete Pipe}

For the next example, we used a $27 \mathrm{~m}$ long concrete pipe. The pipe was made up of 12 sections, with
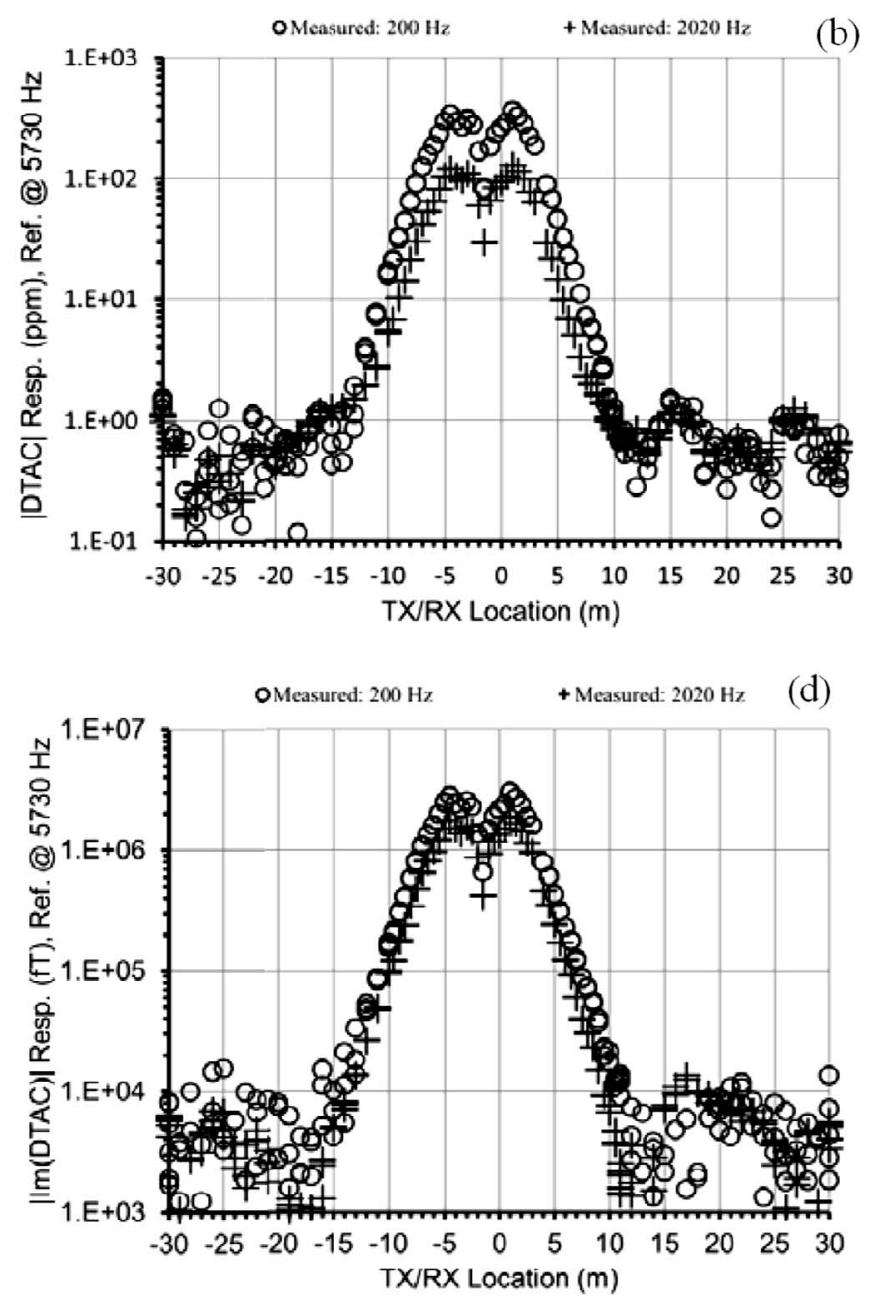

Figure 7. DTAC profile over long concrete pipe. The reference frequency is $5,730 \mathrm{~Hz}$ and data frequencies are $200 \mathrm{~Hz}$ and $2,020 \mathrm{~Hz}$. a) DTAC magnitude in fT; b) DTAC magnitude in ppm; c) real DTAC responses in fT; d) imaginary DTAC responses in $\mathrm{fT}$. 


\section{Feng and Sternberg: DTAC System Field Tests}

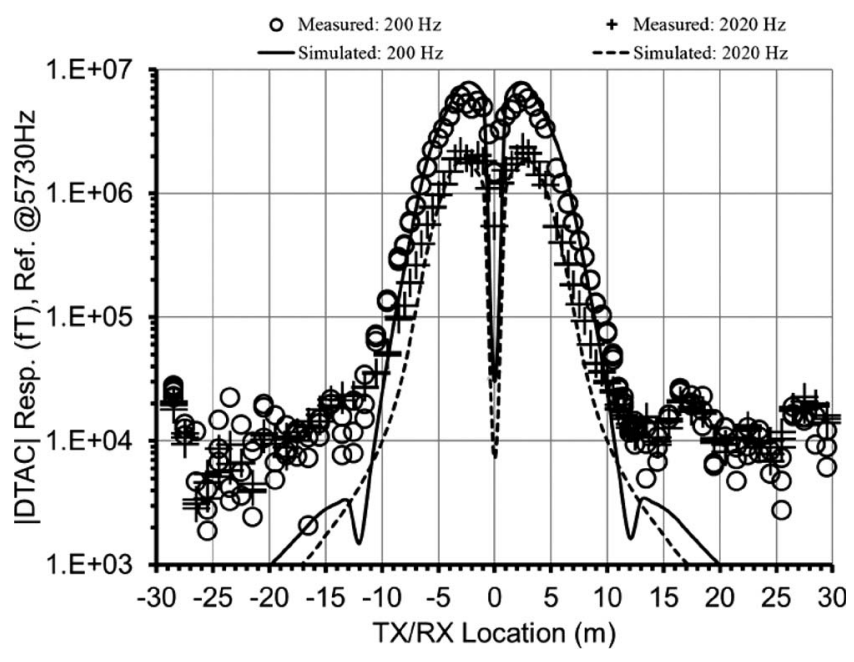

Figure 8. Long concrete pipe profile with theoretical calculation (line) for EMIGMA ILN prism response.

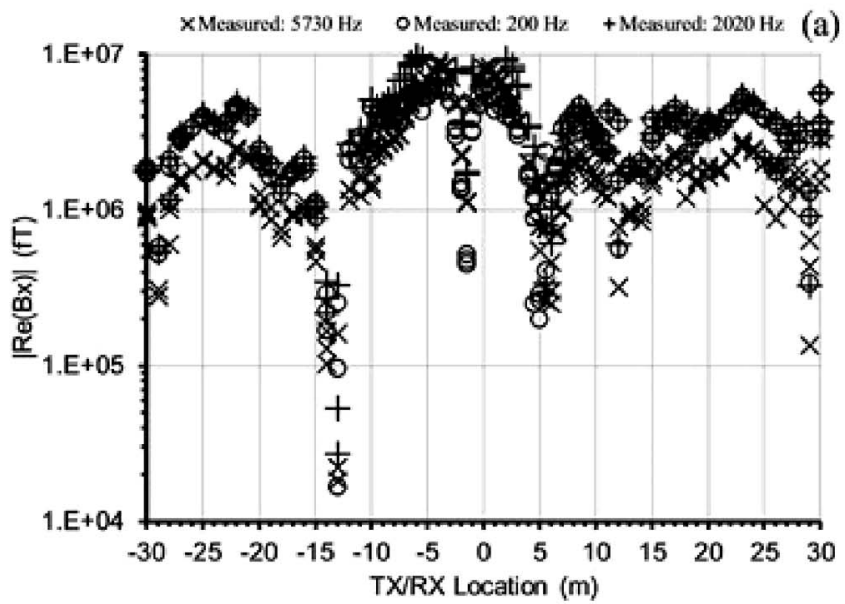

each section $2.25 \mathrm{~m}$ long, $1.2 \mathrm{~m}$ inside diameter, and 1.5 $\mathrm{m}$ outside diameter. There is steel remesh inside each concrete section for required strength. The profile is perpendicular to the pipe and $19 \mathrm{~m}$ from the northern edge of the pipe.

Figure 7 shows the DTAC profile over this long concrete pipe. For this case we have used the $5730 \mathrm{~Hz}$ as the reference frequency and we have measured both 200 $\mathrm{Hz}$ and $2,020 \mathrm{~Hz}$ data frequencies. Once again, there is a very symmetric anomaly, with a background noise of the order of 1,000 to $10,000 \mathrm{fT}$ (or 0.1 to $1 \mathrm{ppm}$ ).

This profile, like the other profiles in this paper, was collected by stopping at each measurement location and collecting three independent one-second-long records. These multiple measurements allow us to estimate the noise level at each location. We also measured the same profile by continuously moving along the profile at a speed of $0.7 \mathrm{~km} / \mathrm{h}(0.4 \mathrm{miles} / \mathrm{h})$. Although not shown,

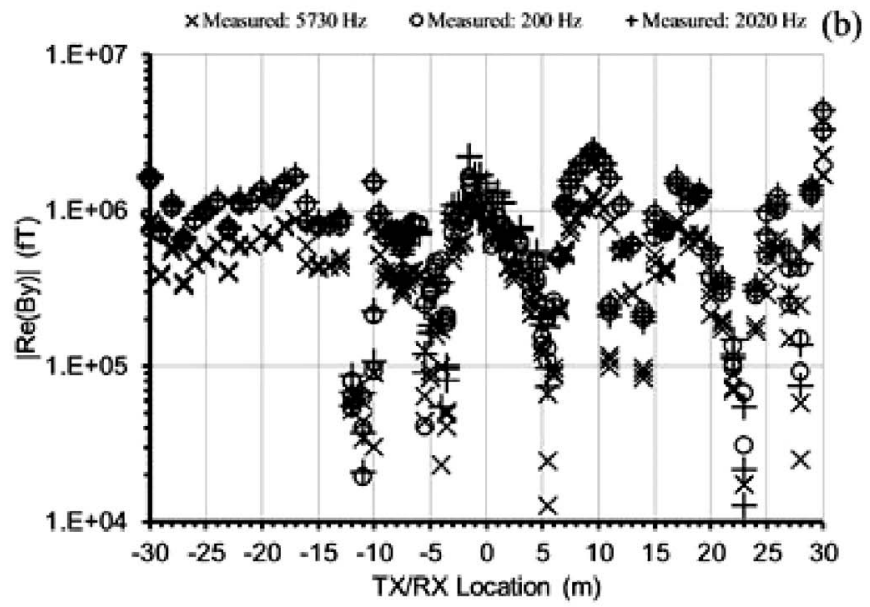

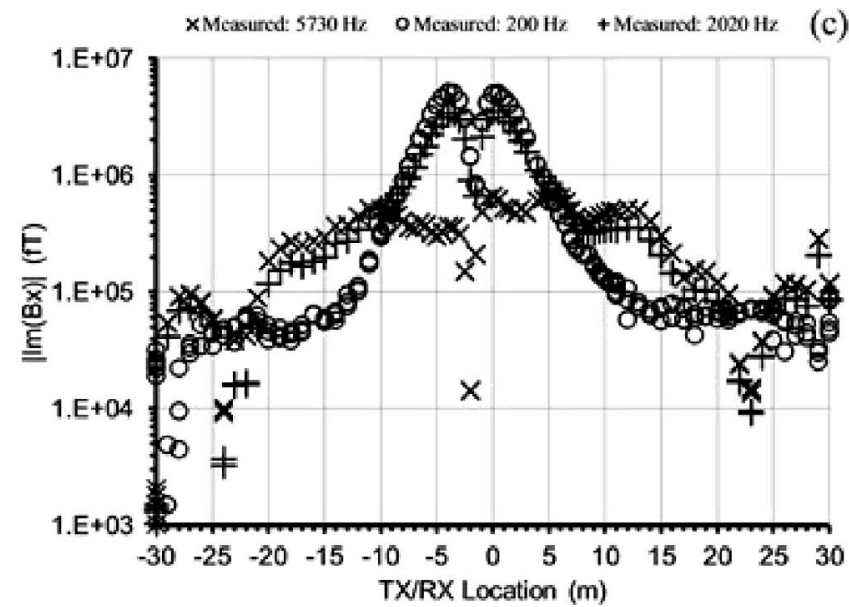

(c)

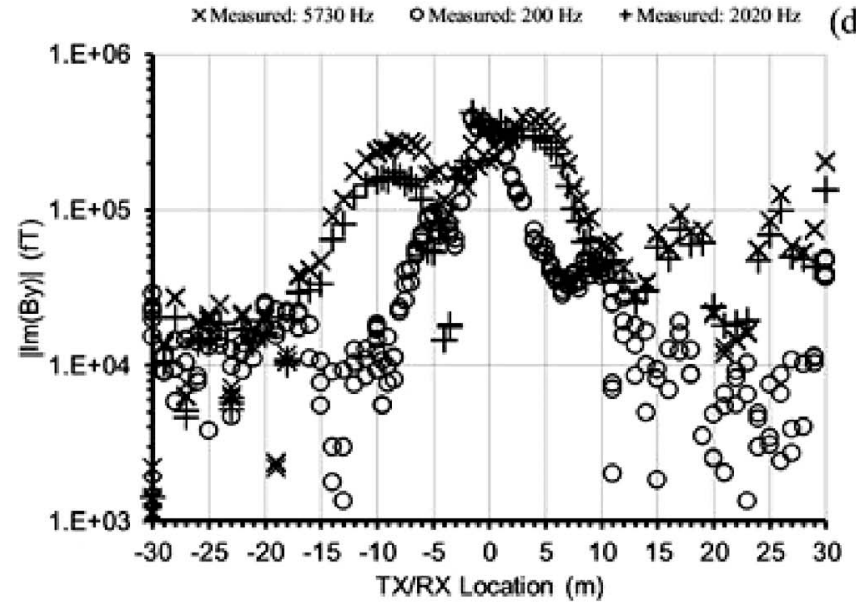

(d)

Figure 9. Conventional $B_{x}$ and $B_{y}$ field data without any DTAC processing for long concrete pipe. a) Real $B_{x}$ magnitude fT; b) Real $B_{y}$ magnitude fT; c) Imaginary $B_{x}$ magnitude in fT; d) Imaginary $B_{y}$ magnitude in fT. 
Journal of Environmental and Engineering Geophysics
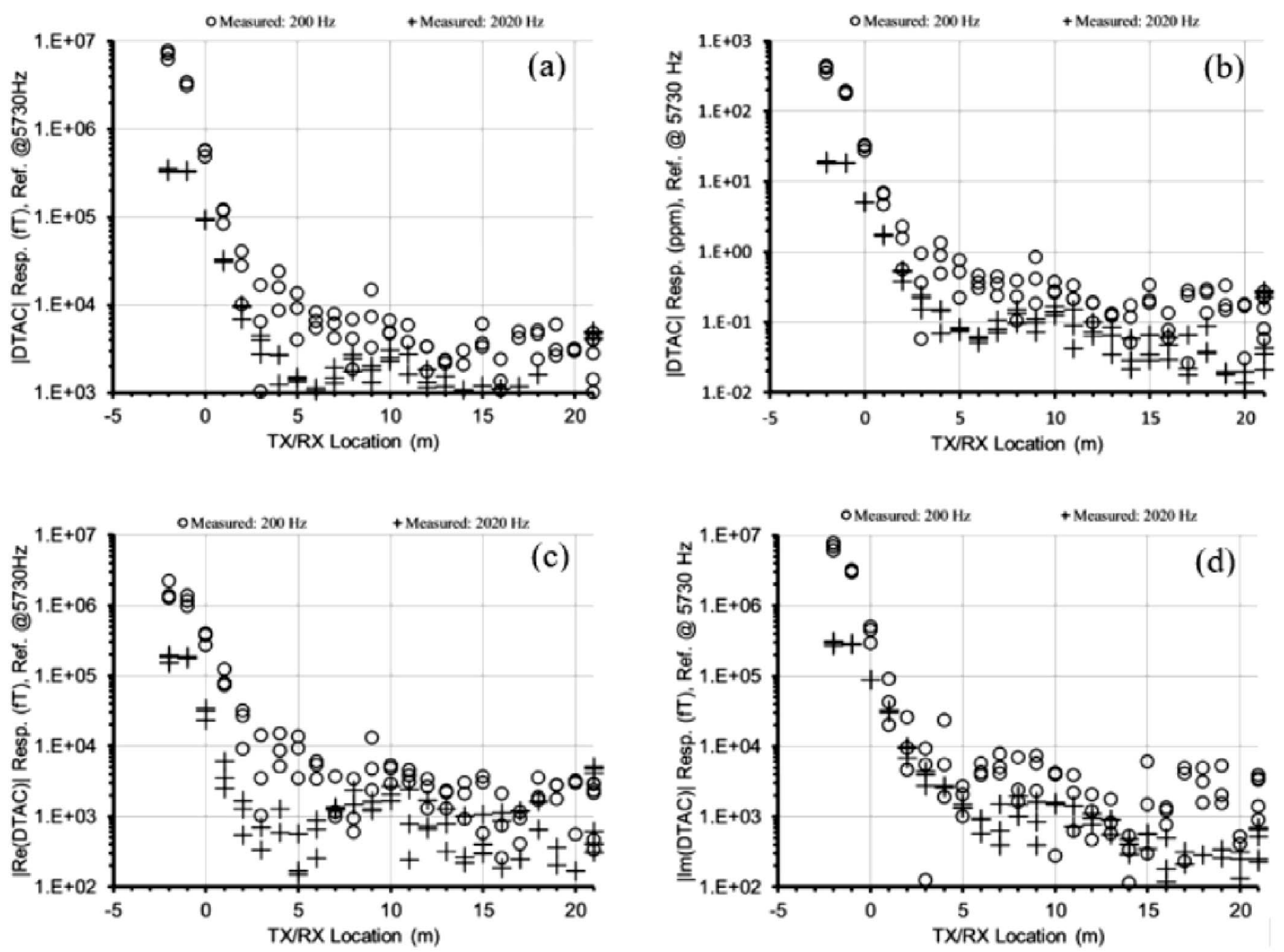

Figure 10. DTAC response from surface interference (automobile). a) DTAC magnitude in fT; b) DTAC magnitude in ppm; c) Real DTAC responses in fT; d) Imaginary DTAC responses in fT.

the measured response with the moving system is very similar to the start/stop response that is shown in Fig. 7.

Figure 8 shows a comparison of the measured data and the calculated EMIGMA model response. For the EMIGMA calculation, we have used a prism model and the ILN algorithm, with a background resistivity of 50 Ohm-m, the prism has a cross-section area equal to the concrete pipe area, with a conductivity of $1,000 \mathrm{~S} / \mathrm{m}$. We note that the $1,000 \mathrm{~S} / \mathrm{m}$ value is a composite value for the steel remesh in the concrete pipe and the air-filled cavity inside the pipe. We found that the EMIGMA calculation cannot handle very long models well. Models that used the actual pipe length of $27 \mathrm{~m}$, or longer lengths, led to errors in the calculated response. However, for the current TX loop size, and for this type of model, all lengths between about $9 \mathrm{~m}$ and $18 \mathrm{~m}$ produced substantially the same DTAC response, which also matched well the observed DTAC response. For shorter prism lengths, the DTAC response does depend on the target length. For a very long target, a limitedlength prism produces an equivalent anomaly to the very long target, and can accurately model the response from this very long target. Using this modeling approach, there is a very close match between the measured response and the theoretical model response for this case.

For comparison, in Fig. 9 we show the raw measured real and imaginary fields for both the $B_{x}$ and $\mathrm{B}_{\mathrm{y}}$ coils. Because of small variations in the primary-field coupling, the real response has wild variations along the profile, with little or no information about the presence of a target. The imaginary response contains some useful information, but has too high a background level and an erratic target response at some frequencies. A careful 
Feng and Sternberg: DTAC System Field Tests
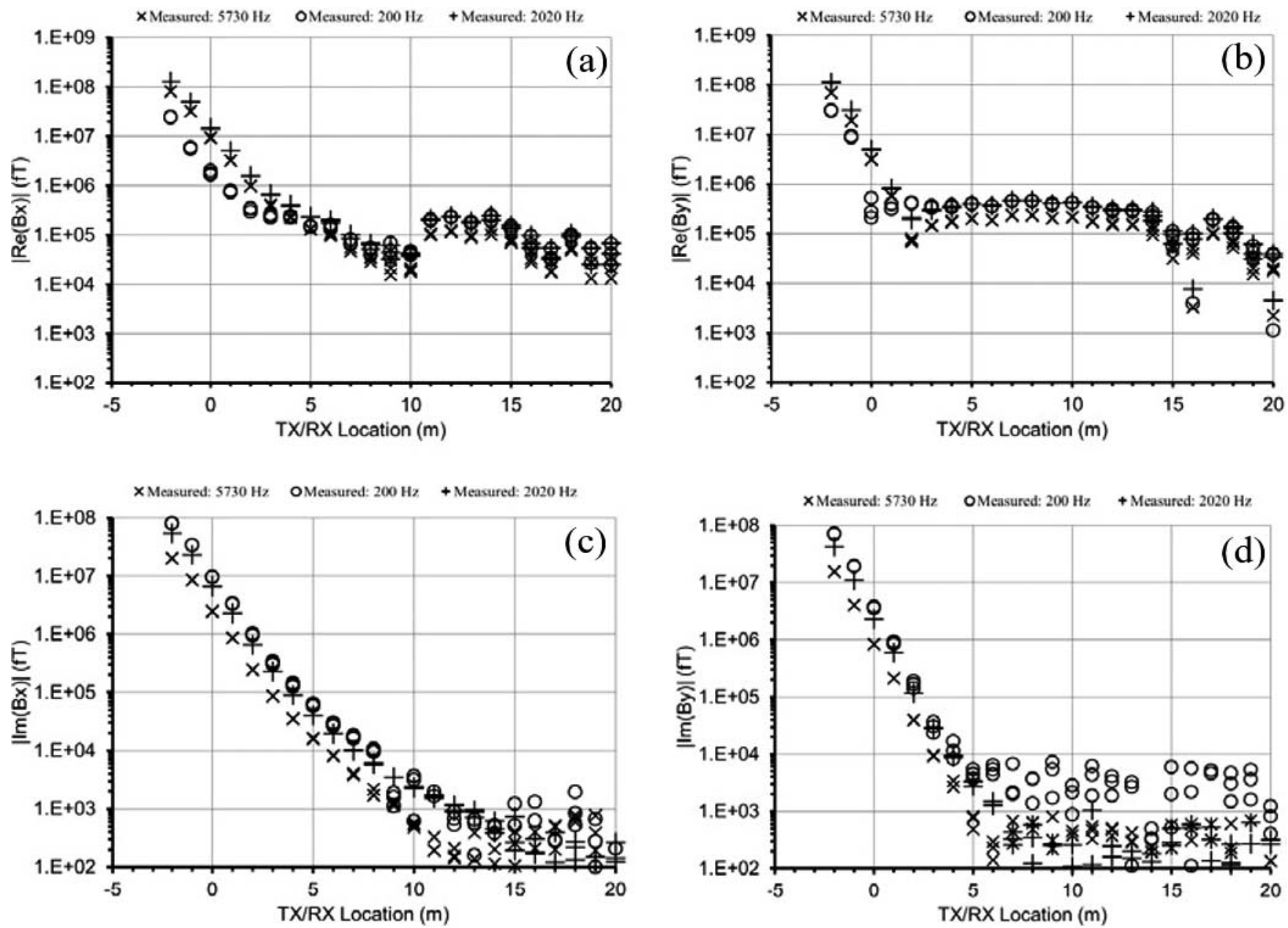

Figure 11. Conventional $B_{x}$ and $B_{y}$ field data without any DTAC processing for surface interference. a) Real $B_{x}$ magnitude $f T$; b) Real $B_{y}$ magnitude $f T$; c) Imaginary $B_{x}$ magnitude in $f T$; d) Imaginary $B_{y}$ magnitude in $f T$.

comparison of Fig. 9 with Fig. 8 shows the significant benefits of the DTAC measuring system

\section{DTAC Response from Surface Interference (Auto- mobile)}

The final example that we will show is for the DTAC response from surface interference. We have used a Honda CRV automobile for this test. For this test only, the frame is completely stationary and the automobile was moved; we have therefore removed all background fields at this point. Since the frame never moves, there is reduced variation in the primary field. Therefore, we are able to utilize both the real and imaginary, $\mathrm{B}_{\mathrm{x}}$ and $\mathrm{B}_{\mathrm{y}}$ fields in this stationary frame test.

Figure 10 shows the DTAC response. The distance on the plots is the separation between TX loop edge and the front edge of the vehicle. The negative distance means the front edge of vehicle has passed beneath the edge of the TX loop. The vehicle has no significant effect on the DTAC response for measurements at distances greater than about $2 \mathrm{~m}$, in other words, the DTAC response is below the naturally occurring noise level past about $2 \mathrm{~m}$. In Fig. 11, we show the real and imaginary, $\mathrm{B}_{\mathrm{x}}$ and $\mathrm{B}_{\mathrm{y}}$ responses. These responses extend out to as far as $15 \mathrm{~m}$ for the $\mathrm{B}_{\mathrm{x}}$ field and out to at least 5 $\mathrm{m}$ for the be $\mathrm{B}_{\mathrm{y}}$ field. In this test, because the vehicle was approaching the frame along the $\mathrm{x}$-axis, with the vehicle center line offset $2 \mathrm{~m}$ from the frame center line, the responses of the $B_{x}$ fields extend out further than that of the $\mathrm{B}_{\mathrm{y}}$ fields.

The DTAC system is relatively insensitive to surface clutter because the magnetic fields induced in the conductive surface clutter are primarily frequency independent, and therefore produce a small DTAC response. This is fundamentally different from the response to buried targets, where the current induced in the background earth is channeled into or around the 


\section{Journal of Environmental and Engineering Geophysics}

target and produces a frequency-dependent response, and therefore a significant DTAC anomaly. A detailed analysis of this is given in Dvorak and Sternberg (2013). The relative insensitivity to surface clutter, (i.e., metal above the ground surface) is one of the main strengths of the DTAC method. The DTAC method is also able to operate relatively closely to interference from other surface clutter, such as fences and power lines.

\section{Conclusions}

Tests using known targets have effectively demonstrated the capabilities of the DTAC method for subsurface imaging. Our future plans are to apply the DTAC method to a wide variety of applications, including civil engineering, water resources, environmental characterization, mining, and other naturalresource exploration. We also anticipate adapting the DTAC system for airborne surveys, using a helicopter. We note that the depth of investigation can be extended far beyond the shallow known targets that we have looked at in this paper. We believe that by scaling up the transmitter moment, this vertical-array method can achieve a depth of investigation of at least several hundred meters.

\section{Acknowledgements}

We would like to thank our colleague, Dr. Steven L. Dvorak, for his many contributions during the previous stages of development of the DTAC method. We would also like to thank Jacob Sheehan and an anonymous reviewer for their very helpful comments and Dale Rucker for his editorial work.

\section{References}

Dvorak, S.L., and Sternberg, B.K., 2002, Removal of time-varying errors in network analyzer measurements: Signal normalization and test results: IEEE Proc. Science Measurement and Technology, 149, 31-36.

Dvorak, S.L., and Sternberg, B.K., 2013, Analytical and numerical studies of a differential target antenna coupling method for imaging subsurface targets: Journal of Environmental and Engineering Geophysics, 18, 91-101.

Dvorak, S.L., and Sternberg, B.K., 2014, Analytical studies of the vertical array differential target antenna coupling (DTAC) method for rapid sensing and imaging of subsurface targets: Journal of Applied Geophysics, 105, $34-49$.

Dvorak, S.L., Sternberg, B.K., and Feng, W., 2017, Design and verification of wide-band, simultaneous, multi-frequency, tuning circuits for large moment transmitter loops: Journal of Applied Geophysics, 138, 172-178.

EMIGMA, 2016, PetRos Eikon, http:/www.petroseikon.com/ emigma/.

Murray, I.R., 1997, On extending the localized non-linear approximation to inductive modes: in Extended Abstracts: 59th European Association of Geoscientists and Engineers, F004.

Murray, I.R., Alvarez, C., and Groom, R.W., 1999, Modelling of complex electromagnetic targets using advanced non-linear approximator techniques: in Expanded Abstracts of the 69th Annual International Meeting, Society of Exploration Geophysicists, 271-274.

Sternberg, B.K., and Dvorak, S.L., 2002, Removal of time-varying errors in network analyzer measurements: System design: IEEE Proc. Science Measurement and Technology, 149, 22-30.

Sternberg, B.K., and Dvorak, S.L., 2013, Experimental studies and verification of a Differential Target Antenna Coupling method for sensing and imaging subsurface targets: Journal of Environmental and Engineering Geophysics, 18, 91-101.

Sternberg, B.K., Feng, W., Dvorak, S.L., and Reavis, R.D., 2015a, Experimental studies and verification of the vertical array, Differential Target Antenna Coupling (DTAC) method for rapid sensing and imaging of subsurface targets: Journal of Environmental and Engineering Geophysics, 20, 119-136.

Sternberg, B.K., Feng, W., and Dvorak, S.L., 2015b, Numerical studies and potential applications of the vertical array, Differential Target Antenna Coupling (DTAC) method for rapid sensing and imaging of subsurface targets: Journal of Environmental and Engineering Geophysics, 20, 137151.

Sternberg, B.K., Dvorak, S.L., and Feng, W., 2017, Design and verification of large moment transmitter loops for geophysical applications, Journal of Applied Geophysics, 136, 211218.

Walker, P.W., and West, G.F., 1991. A robust integral equation solution for electromagnetic scattering by a thin plate in conductive media: Geophysics, 56, 1140-1152. 